\title{
A systematic review of the use of an expertise-based randomised controlled trial design
}

Jonathan A. Cook ${ }^{1 *}$, Andrew Elders², Charles Boachie ${ }^{2}$, Ted Bassinga ${ }^{2}$, Cynthia Fraser $^{2}$, Doug G. Altman ${ }^{1}$, Isabelle Boutron ${ }^{3}$, Craig R. Ramsay ${ }^{2}$ and Graeme S. MacLennan ${ }^{2}$

\begin{abstract}
Background: Under a conventional two-arm randomised trial design, participants are allocated to an intervention and participating health professionals are expected to deliver both interventions. However, health professionals often have differing levels of expertise in a skill-based interventions such as surgery or psychotherapy. An expertise-based approach to trial design, where health professionals only deliver an intervention in which they have expertise, has been proposed as an alternative. The aim of this project was to systematically review the use of an expertise-based trial design in the medical literature.
\end{abstract}

Methods: We carried out a comprehensive search of nine databases_-AMED, BIOSIS, CENTRAL, CINAHL, Cochrane Methodology Register, EMBASE, MEDLINE, Science Citation Index, and PsycINFO_from 1966 to 2012 and performed citation searches using the ISI Citation Indexes and Scopus. Studies that used an expertise-based trial design were included. Two review authors independently screened the titles and abstracts and assessed full-text reports. Data were extracted and summarised on the study characteristics, general and expertise-specific study methodology, and conduct.

Results: In total, 7476 titles and abstracts were identified, leading to 43 included studies (54 articles). The vast majority (88 \%) used a pure expertise-based design; three (7\%) adopted a hybrid design, and two (5\%) used a design that was unclear. Most studies compared substantially different interventions (79 \%). In many cases, key information relating to the expertise-based design was absent; only $12(28 \%)$ reported criteria for delivering both interventions. Most studies recruited the target sample size or very close to it (median of 101, interquartile range of 94 to 118), although the target was reported for only $40 \%$ of studies. The proportion of participants who received the allocated intervention was high ( $92 \%$, interquartile range of 82 to $99 \%$ ).

Conclusions: While use of an expertise-based trial design is growing, it remains uncommon. Reporting of study methodology and, particularly, expertise-related methodology was poor. Empirical evidence provided some support for purported benefits such as high levels of recruitment and compliance with allocation. An expertise-based trial design should be considered but its value seems context-specific, particularly when interventions differ substantially or interventions are typically delivered by different health professionals.

Keywords: Expertise-based, Expertise, Systematic review, Learning, Randomised controlled trial, Trial design, Non-pharmacological interventions, Surgery

\footnotetext{
*Correspondence: jonathan.cook@ndorms.ox.ac.uk

${ }^{1}$ Centre for Statistics in Medicine, Nuffield Department of Orthopaedics,

Rheumatology and Musculoskeletal Sciences, Botnar Research Centre,

University of Oxford, Nuffield Orthopaedic Centre, Windmill Road, Oxford

OX3 7LD, UK

Full list of author information is available at the end of the article
}

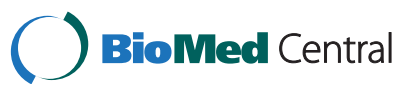

(c) 2015 Cook et al. This is an Open Access article distributed under the terms of the Creative Commons Attribution License (http://creativecommons.org/licenses/by/4.0), which permits unrestricted use, distribution, and reproduction in any medium, provided the original work is properly credited. The Creative Commons Public Domain Dedication waiver (http:// creativecommons.org/publicdomain/zero/1.0/) applies to the data made available in this article, unless otherwise stated. 


\section{Background}

Under a conventional two-arm randomised controlled trial (RCT) (within-health professional) design, participants are randomly allocated to one of the two interventions and all participating health professionals are expected to deliver both interventions. However, if the interventions are skill-based, and particularly when those under evaluation differ substantially, health professionals may (for example, surgeons or therapists) may have differing levels of expertise in the interventions, conduct only one routinely, or have a preference for one over another. These potential obstacles to the conduct of an RCT have been noted particularly in surgery; many surgeons may accept the need in principle for an RCT but may struggle to reconcile their personal involvement with their surgical experience and routine practice [1]. As a result, some may be reluctant to participate in a standard, individually randomised RCT. An expertisebased approach to trial design, in which participating surgeons provide only the intervention in which they have expertise, has been proposed to overcome this problem [2]. As with the within-health professional design, a patient is randomly allocated to an intervention, the difference being that there are two sets of participating health professionals, one for each intervention, who will perform the allocated intervention. Purported benefits of this design include increased surgical participation, recruitment, and compliance with randomisation, addressing the learning curve effect as well as the desirability from the perspective of the patient [2]. Although such a design is not new [3], the profile of the design has been raised and its use appears to becoming more common. For some comparisons such as surgery versus medical management, it has become the default design. However, expertise-based designs have been criticized on a number of grounds [4], including a number of methodological considerations (for example, impact on sample size). In particular, how 'expertise' should be defined is uncertain but clearly is of critical importance. The optimal approach to implementation is also unclear $[1,5]$. It may be that the expertise-based design is most suited to certain research questions [6-12].

Although an expertise-based design has recently received the most attention for surgical evaluation $[1,2]$, it has been used in other areas (for example, psychotherapy) [12]. The same issues exist for the delivery of other interventions which are substantially skill-based and in which individual practitioners may be familiar with only a particular approach or have a strong preference for one intervention over another. However, there is uncertainty regarding under which circumstances an expertisebased trial design is appropriate, how such studies are implemented in practice, and whether the purported benefits have been realised (for example, achieving recruitment and high compliance with intervention). No comprehensive review of expertise-based trials has been previously carried out. A limited search was conducted previously (160 citations) [2] but was restricted to surgery and extended only up to October 2003. Within the small number of studies identified in that search, variation in reporting and practice highlighted the need for both a comprehensive search strategy and an evaluation of differences in how such studies were carried out. For example, different terms have been used to represent the same design (for example, randomised-surgeon design). Beyond the surgical area, other terms (for example, hierarchical and nested) have been used to describe the same design [12]. Within those that might be characterised as an expertise-based design, there are variations in how 'expertise' was defined (for example, surgeon self-certification versus objective requirements) and how health professionals were assigned to a treatment group (health professional preference or randomisation). The aim of this review was to systematically review the use of an expertise-based trial design in the medical literature in order to improve understanding of the applicability, implementation, and implications of using such a design so as to aid the design of future trials. To achieve this, the objectives of the review were (i) to identify the contexts in which an expertise-based trial design has been used, (ii) to assess the methodological variation in expertise-based trial design implementation both in general and those aspects specifically related to the expertise-based design, and (iii) to summarise the reported experience of using such a design.

\section{Methods}

The study protocol is available from the authors. Ethical approval was not required for this research given its focus and given that only publically available information was used.

\section{Inclusion criteria}

RCTs of two medical interventions that used an expertise-based trial (either full or hybrid) design were included (that is, those that used a randomised allocation of participants to skill-based interventions that were delivered by non-overlapping groups of interventionalists). The following types of studies were not eligible for inclusion: those that had been published only as protocols or abstracts, trials with three or more intervention arms, and trials in which the full-text reports were published only in a non-English language journal. Systematic (or narrative) reviews of expertise-based trials were not eligible, but the included studies were assessed for inclusion. No restrictions on the age or type of participants in the trials were made. 


\section{Search}

It was anticipated, from reviewing the results of a scoping search, that it would be difficult to identify all relevant studies from searching bibliographic databases alone given the poor reporting of methods in the titles and abstracts and the paucity of specific controlled vocabulary terms in the major bibliographic databases. To address this, a range of methods were used to retrieve reports of relevant trials. The databases searched included Medical Literature Analysis and Retrieval System Online (MEDLINE), Excerpta Medica dataBASE (EMBASE), Cumulative Index to Nursing and Allied Health Literature (CINAHL), BioSciences Information Service of Biological Abstracts (BIOSIS), Cochrane Central Register of Controlled Trials (CENTRAL), Cochrane Methodology Register, Science Citation Index, The Allied and Complementary Medicine Database (AMED), and PsycINFO. In addition, cited reference searches (using Institute for Scientific Information Citation Indexes and Scopus), references of included articles, and key author searches were undertaken. If a study had been referred to as an expertise-based trial in another report but the design was unclear in the related published literature, an investigator was contacted to confirm. Searches were undertaken from 1966 onwards or, from the start of database coverage if after 1966. The MEDLINE and EMBASE multi-file search strategy, which was used to search these databases concurrently, was developed first and translated for other databases. The search strategies are provided in Additional file 1.

\section{Selection of studies}

Two review authors (JC and CB, AE, GSM, or TB) independently screened the titles and abstracts of all reports identified by the search strategy. The full versions of articles not definitely excluded at that stage were obtained for full-text assessment, which will be carried out by two reviewers as before. Where they occurred, differences of opinion were discussed; if necessary, a blinded third review author acted as arbitrator. If appropriate, a clinical area expert was consulted. Methods articles were not included but were set aside for future reference and, if appropriate to do so, were considered in the discussion.

\section{Data collection and analysis}

Data on the trial characteristics and context (year of publication, funding [13], clinical area, interventions under evaluation, and type of comparison), expertisebased design methodology and related reporting (type of design, reporting including stated advantages and disadvantages, interventionalist mechanism of allocation, eligibility criteria, and number), general study methodology and conduct (randomisation, blinding, and study size and compliance regarding allocation), and details on the primary outcome and statistical analyses were extracted. One reviewer (JC) carried out data extraction of included articles (using a pro forma specifically designed for the purpose). A second reviewer (AE or GM) independently assessed a random sample of $25 \%$ of included studies. Data were summarised. Owing to the relatively small number of included studies, a planned statistical analysis to compare factors across trial types was not carried out.

\section{Results}

In total, 7476 titles and abstracts were identified from the search of 9 databases (Fig. 1); after removal of duplicates, 3247 were screened for inclusion. Of these, 3092 were excluded and 155 articles were selected for full-text assessment. A further 19 articles were identified from the references of included studies, leading to a total of 157 studies (174 articles) that were full-text-assessed. Of these, 117 studies (125 articles) were excluded, leaving 40 studies (49 articles) that, combined with three studies (five articles) identified from a published review, led to 43 studies (54 articles) in total. The list of included studies is provided in Additional file 2. Table 1 shows the study characteristics and context of the included studies. The earliest publication was in 1982; most (69 \%) were published from 2000 onwards. The vast majority received funding from public bodies in whole or part, whereas partial or full funding was received less commonly from a charity or a commercial source. The clinical areas represented varied, and mental health (26\%) and musculoskeletal (19\%) were the most common. The most common intervention types were a procedure and psychotherapy (40 and $37 \%$ for interventions 1 and 2 for both). The comparisons overwhelmingly focussed on substantial differences between interventions (79\%).

Table 2 reported the methodology and reporting relating to the expertise-based trial design. The vast majority (88 \%) used a pure expertise-based design in which health professionals deliver only one of the two interventions; only three (7 \%) used a hybrid designin which some health professionals could deliver both. For two studies the design that was unclear (5\%). Ten used terminology to refer to the design, and six different names were used. The level of detail reported in the abstract varied from stating the design name in six (14\%) studies to giving no details in 15 (35\%). A variety of advantages of using an expert-based design were stated: the most common were ensuring a balance of health professionals in terms of interest and commitment (9\%), addressing learning (7\%), ensuring that the intervention is delivered by someone with expertise in order to avoid criticism (5\%), and reducing cross-over (5\%). Fewer disadvantages were stated; the more common were that intervention deliverers were not representative of clinical practice or may 


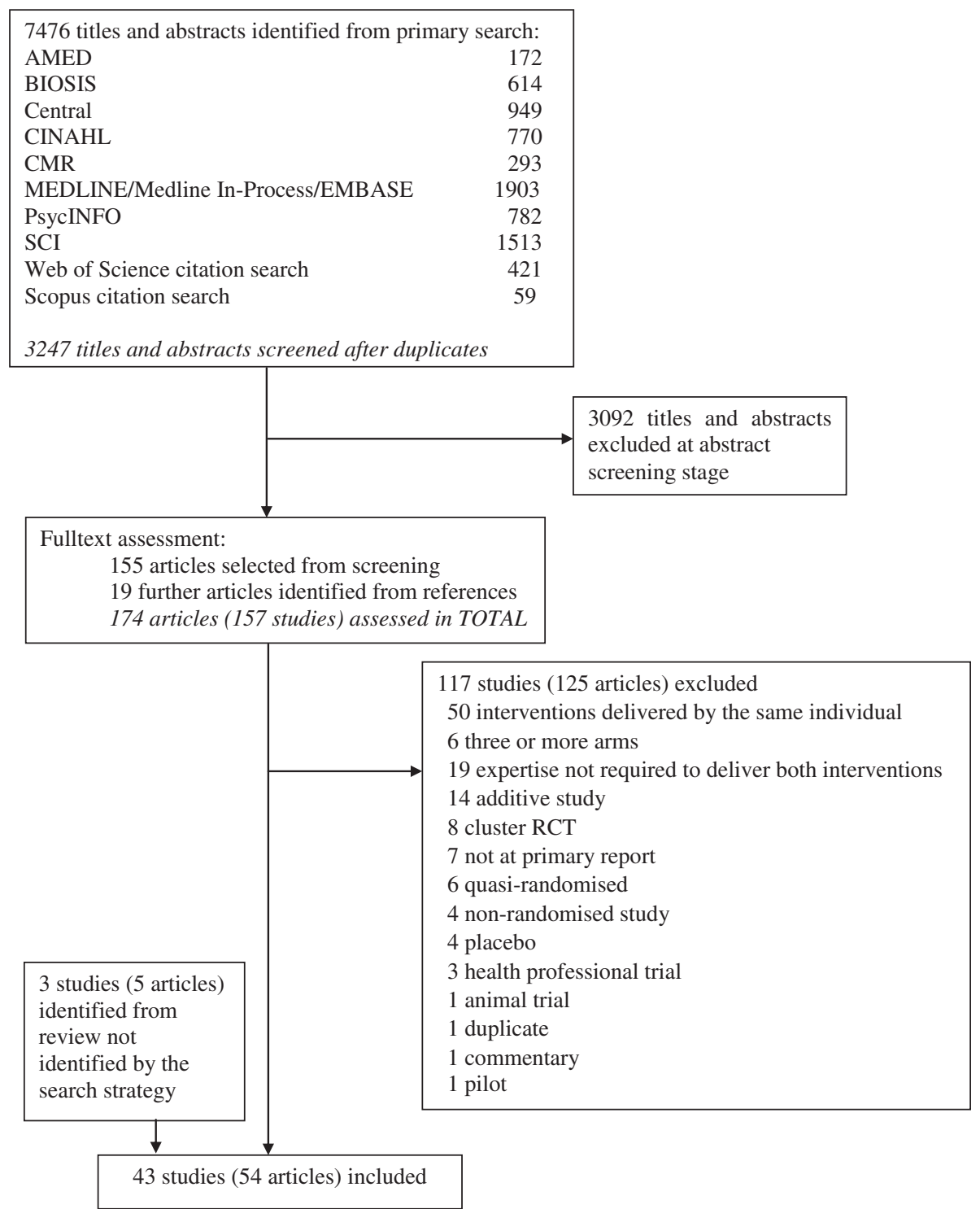

Fig. 1 PRISMA Flow diagram. AMED, The Allied and Complementary Medicine Database; BIOSIS, BioSciences Information Service of Biological Abstracts; CENTRAL, Cochrane Central Register of Controlled Trials; CINAHL, Cumulative Index to Nursing and Allied Health Literature; CMR, Cochrane Methodology Register; EMBASE, Excerpta Medica dataBASE; MEDLINE, Medical Literature Analysis and Retrieval System Online; RCT, randomised controlled trial; SCl, Science Citation Index

not be balanced between groups unless selected $(9 \%)$ and that the delivery of interventions may vary between groups beyond the allocated intervention (5\%). Under half reported the method of allocation of health professoinal to intervention group; eight (19\%) interestingly used randomisation, five (12\%) followed usual practice, four followed interventionalist preference $(9 \%)$, and in one case $(2 \%)$ the type of health professional was defined as part of the research question. Criteria for delivering an intervention varied between groups and involved one or more of health professional training/grades, years of experience in and/or number of cases of delivering the intervention, prior training and/or supervision in using the intervention, specified outcome performance level, recommendation by colleague, experience of working with patient group, and willingness to learn a new procedure. Only $12(28 \%)$ stated the criteria for delivering both interventions. A substantial number $(21,49 \%)$ did not give the number of health professionals delivering the interventions for both groups. The number of health professionals was similar for the two arms (medians of 6 and 5 , respectively).

Details regarding general study methodology and conduct are shown in Table 3. Reporting of participant randomisation methodology was adequate for the majority of studies (58 and $60 \%$ for sequence generation and 
Table 1 General study characteristics and context ( $n=43$ unless otherwise stated)

\begin{tabular}{|c|c|c|}
\hline & Number & Percentage \\
\hline \multicolumn{3}{|l|}{ Year of publication } \\
\hline 1980-1989 & 2 & 5 \\
\hline 1990-1999 & 11 & 26 \\
\hline 2000-2009 & 23 & 53 \\
\hline 2010-2012 & 7 & 16 \\
\hline \multicolumn{3}{|l|}{ Funding $(n=28)$} \\
\hline Charity & 3 & 11 \\
\hline Commercial & 1 & 4 \\
\hline Public body & 14 & 50 \\
\hline Mixed & 10 & 37 \\
\hline \multicolumn{3}{|l|}{ Clinical area } \\
\hline Cardiology & 5 & 5 \\
\hline Emergency care & 1 & 2 \\
\hline Gastroenterology & 2 & 5 \\
\hline General medicine & 4 & 9 \\
\hline Geriatrics & 1 & 5 \\
\hline Mental health & 11 & 26 \\
\hline Musculoskeletal & 8 & 19 \\
\hline Neurology & 3 & 7 \\
\hline Obstetrics and gynaecology & 2 & 5 \\
\hline Oncology & 1 & 2 \\
\hline Ophthalmology & 1 & 2 \\
\hline Substance abuse & 4 & 5 \\
\hline \multicolumn{3}{|l|}{ Intervention 1} \\
\hline Acupuncture & 1 & 2 \\
\hline Chiropractic & 2 & 5 \\
\hline $\begin{array}{l}\text { Clinical management } \\
\text { (various) }\end{array}$ & 1 & 2 \\
\hline Physiotherapy & 3 & 7 \\
\hline Procedure & 17 & 40 \\
\hline Psychotherapy & 17 & 40 \\
\hline $\begin{array}{l}\text { Rehabilitation management } \\
\text { (various) }\end{array}$ & 2 & 5 \\
\hline \multicolumn{3}{|l|}{ Intervention 2} \\
\hline Anaesthetic & 1 & 2 \\
\hline Chiropractic & 1 & 2 \\
\hline $\begin{array}{l}\text { Clinical management } \\
\text { (various) }\end{array}$ & 3 & 7 \\
\hline $\begin{array}{l}\text { Clinical management } \\
\text { (various)/procedure }\end{array}$ & 1 & 2 \\
\hline Physiotherapy & 3 & 7 \\
\hline Procedure & 16 & 37 \\
\hline Psychotherapy & 16 & 37 \\
\hline Rehabilitation management & 2 & 5 \\
\hline
\end{tabular}

Table 1 General study characteristics and context ( $n=43$ unless otherwise stated) (Continued)

\begin{tabular}{lll}
\hline Difference between interventions & & \\
Minor & 8 & 19 \\
Substantial & 34 & 79 \\
Different type of care & 1 & 2 \\
\hline
\end{tabular}

allocation concealment, respectively). Blinding of the outcome assessment was known to be performed in 12 (28 \%) studies, but blinding of participants was known to be performed in only one ( $2 \%)$. Most studies were single-centre RCTs (median of 1 , interquartile range (IQR) of 1 to 5). Study size was typically moderate (111, IQR of 62 to 226). Most studies recruited the target number or very close to it (101, IQR of 94 to 118$)$, although the target size was reported for only $40 \%$ of studies. The proportion of participants who received the allocated intervention was high (92\%, IQR of 82 to $99 \%)$. For those that reported this aspect, occurrences of cross-over between interventions or receiving a 'third' (non-trial) intervention were rare. Table 4 reports details on the primary outcome. The most common outcomes were type of disease and specific quality of life (29\%) followed by other patient-reported outcomes and process measure (17 \% for both). The number of observations available to analyse was poorly reported (26 studies, or $60 \%)$. Details regarding the statistical analysis carried out are provided in Table 5. Almost all studies (98\%) carried out an analysis by randomised groups. Eight (19\%) studies had a compliance analysis, and only two (5\%) adjusted for clustering of outcome by interventionalists.

\section{Discussion}

This systematic review identified from across the medical literature a number of RCTs that used an expertisebased trial design. An expertise-based design differs from the conventional (within-health professional) design by allowing different groups of health professionals to deliverer the interventions under evaluation as opposed to requiring participating health professionals to deliver both of (or all, where there are three or more) the interventions. It has been suggested that the design is able to address $[2,3]$ some of the challenges of conducting an RCT of skill-based interventions which a conventional design is not. Although the design has been in use for over 30 years and use has been growing in recent years, it remains uncommon.

Previous limited reviews [2, 14] had identified fewer than 10 studies using an expertise-based design and focussed only on surgery. Uniquely, this review had a full and comprehensive systematic search with information on the context of the study, the methodology of the trial 
Table 2 Expertise-based design methodology and related reporting ( $n=43$ unless otherwise stated)

Number Percentage

Expertise-based design type

Pure

Hybrid

Unclear

$3 \quad 7$

Name used $(n=24)$

Expertise-based

Double randomisation

Randomised to surgeon

Non-randomised surgeon design

Randomised-surgeon

Surgeon-randomised

None

25

Reporting of expertise-based design in abstract

Design name

Deliverers of interventions stated to be different

Details regarding health professionals delivering one intervention

Details regarding health professional delivering both interventions

No details

7

2

2

1

2

15

Reported advantages $(n=20)$

Ensuring intervention was delivered by someone with expertise to avoid criticism of the study

Balance of health professionals (e.g., interest, commitment, and prior knowledge of intervention)

Randomisation of health professional 'consistent with efficacy trial'

Following preference will reduce non-compliance

Using randomisation of health professional strengthens generalisability of findings

Eliminates learning of the intervention

Eliminates ethical concerns with intervention deliverer not doing what they would do outside of the trial

Delivery of intervention maximised (and may reduce adverse events)

Ensures experience in control group

Reduces cross-over between group compared with conventional study

Avoid non-compliance with allocation because of non-familiarity

Health professionals delivering their preferred intervention

Following usual practice reduces non-compliance with allocation

Reported disadvantages $(n=9)$

Health professionals delivering interventions may not be representative of practice

Health professionals delivering interventions may not be balanced (e.g., motivation and prior experience) unless selected

Delivery may vary in other ways between groups because of different health professionals delivering the interventions

Disagreement between recruiter and health professional delivering the intervention regarding eligibility led to the intervention not being performed in some cases

Addition of new intervention and deliverer may create expectation bias

$6 \quad 14$

92

$7 \quad 16$

$6 \quad 14$

$15 \quad 35$

Allocation of intervention deliverers

Randomised

Usual practice

Preference

Defined by research question

8

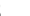

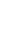


Table 2 Expertise-based design methodology and related reporting ( $n=43$ unless otherwise stated) (Continued)

\begin{tabular}{|c|c|c|}
\hline Not stated & 25 & 58 \\
\hline \multicolumn{3}{|l|}{ Criteria for delivering intervention 1} \\
\hline Number of prior cases & 2 & 4 \\
\hline Number of years of experience and prior cases & 1 & 2 \\
\hline Number of years of experience and training in intervention & 1 & 2 \\
\hline 'Qualified' intervention deliverer & 1 & 2 \\
\hline Training of therapy and group supervision & 1 & 2 \\
\hline Profession qualification & 3 & 6 \\
\hline Prior training and experience of intervention & 1 & 2 \\
\hline Trained in delivering intervention & 4 & 8 \\
\hline Recommendation by colleagues as expert & 1 & 2 \\
\hline Experience of working with patient group & 1 & 2 \\
\hline Willingness to learn new intervention & 1 & 2 \\
\hline Without prior experience of intervention (training then provided) & 1 & 2 \\
\hline None (training/supervision provided as part of the study) & 3 & 7 \\
\hline Not stated & 22 & 51 \\
\hline \multicolumn{3}{|l|}{ Criteria for delivering intervention 2} \\
\hline Number of years of experience and specific outcome levels to be achieved & 1 & 2 \\
\hline Years of experience & 1 & 2 \\
\hline Number of years of experience and prior cases & 1 & 2 \\
\hline Recommendation by colleagues as expert & 1 & 2 \\
\hline Experience of working with patient group & 1 & 2 \\
\hline Professional qualification & 2 & 4 \\
\hline Preference and no training in alternative intervention & 1 & 2 \\
\hline Willingness to learn new intervention & 1 & 2 \\
\hline Interest in patient group & 1 & 2 \\
\hline None (trained as part of the study) & 2 & 5 \\
\hline None stated & 31 & 72 \\
\hline Criteria provided for both intervention 1 and 2 deliverers & 12 & 28 \\
\hline \multicolumn{3}{|l|}{ Number of health professionals delivering intervention 1} \\
\hline Reported & 30 & 69 \\
\hline Median (interquartile range), range & & $1-58$ \\
\hline \multicolumn{3}{|l|}{ Number of health professionals delivering intervention 2} \\
\hline Fully reported & 23 & 53 \\
\hline Median (interquartile range), range & \multicolumn{2}{|c|}{$5(2-19), 1-63$} \\
\hline
\end{tabular}

(both expertise-related and generic), and the reported views of the trialists collected and summarised. It shows that the design has been used beyond surgical interventions where its use has been previously proposed $[2,3,15]$ and covers many clinical areas and evaluations of nonpharmacological interventions. The potential value over a conventional design appears to be case-specific and is clear in two main circumstances. One is where two skillbased interventions that are substantially different from one another are to be compared (for example, cognitive behavioural therapy and limited professional support); this is the situation where they have most commonly been used. Another setting where they have been used and seem particularly appropriate (or perhaps natural to use) is when the interventions are typically delivered by another type of health professional (for example, a comparison of coronary artery bypass graft and stenting for the treatment of coronary artery disease where they are delivered by a surgeon and a cardiologist respectively, as is the case in some countries). Two surveys of preferences regarding trial design showed differing levels of support for an expertise-based design, suggesting that it merits 
Table 3 Study methodology and conduct ( $n=43$ unless otherwise stated)

\begin{tabular}{|c|c|c|}
\hline & Number & Percentage \\
\hline \multicolumn{3}{|c|}{ Intervention deliverers-randomisation sequence generation } \\
\hline Adequate & 1 & 10 \\
\hline Inadequate & 0 & 0 \\
\hline Unclear & 9 & 90 \\
\hline \multicolumn{3}{|c|}{ Intervention deliverers-randomisation allocation concealment } \\
\hline Adequate & 1 & 10 \\
\hline Inadequate & 0 & 0 \\
\hline Unclear & 9 & 90 \\
\hline \multicolumn{3}{|c|}{ Participants-randomisation sequence generation } \\
\hline Adequate & 25 & 58 \\
\hline Inadequate & 0 & 0 \\
\hline Unclear & 18 & 42 \\
\hline \multicolumn{3}{|c|}{ Participants-randomisation allocation concealment } \\
\hline Adequate & 26 & 60 \\
\hline Inadequate & 2 & 5 \\
\hline Unclear & 15 & 35 \\
\hline \multicolumn{3}{|c|}{ Blinding-primary outcome assessment } \\
\hline Yes & 12 & 28 \\
\hline No & 27 & 63 \\
\hline Unclear & 4 & 9 \\
\hline \multicolumn{3}{|l|}{ Blinding-participants } \\
\hline Yes & 1 & 2 \\
\hline No & 34 & 79 \\
\hline Unclear & 8 & 19 \\
\hline \multicolumn{3}{|l|}{ Number of centres } \\
\hline Reported & 42 & 98 \\
\hline Median (IQR) & 1 & $(1-5)$ \\
\hline \multicolumn{3}{|l|}{ Study size } \\
\hline Reported & 42 & 98 \\
\hline Median (IQR) & 111 & $(62-226)$ \\
\hline \multicolumn{3}{|c|}{ Percentage of target size recruited } \\
\hline Reported target size & 17 & 40 \\
\hline Median (IQR) & 101 & $(94-118)$ \\
\hline \multicolumn{3}{|c|}{ Percentage received full intervention } \\
\hline Reported & 31 & 74 \\
\hline Median (IQR) & 92 & $(82-99)$ \\
\hline \multicolumn{3}{|c|}{ Cross-over to other study intervention } \\
\hline Reported & 18 & 42 \\
\hline Median (IQR) & 0 & $(0-2)$ \\
\hline \multicolumn{3}{|l|}{ "Third" intervention } \\
\hline Reported & 15 & 35 \\
\hline Median (IQR) & 0 & $(0-0)$ \\
\hline
\end{tabular}

IQR interquartile range consideration with regard to the specific context in which it would be used $[6,9]$.

Reporting of the use of the expertise-based design methodology in the abstract was highly variable, and insufficient details regarding key methodological features of expertise-based trials were provided. Around half failed to provide details on the interventionalists delivering the interventions, a key piece of information for interpreting and assessing the applicability of the findings. Furthermore, we identified the use of a hybrid approach in a small number of studies, although it is possible that more studies adopted this design, but this information was not provided in the trial reports $[5,16]$. Expertise-based trials were typically single-centre studies of moderate size with around 10 interventionalists involved in the study with a patient-reported primary outcome. Two of the main purported benefits appeared to be supported by the empirical evidence. Achievement of the target recruitment was very high, although the target size was often not reported, leading to some uncertainty regarding this finding. However, compliance with the allocated intervention was generally very high (median of at least $90 \%$ ), and occurrences of cross-over between intervention groups or receiving a third nontrial intervention were rare.

Table 4 Primary outcome(s) ( $n=43$ unless otherwise stated)

\begin{tabular}{|c|c|c|}
\hline & Number & Percentage \\
\hline \multicolumn{3}{|l|}{ Primary outcome } \\
\hline \multicolumn{3}{|l|}{ Number of primary outcomes } \\
\hline None & 17 & 40 \\
\hline 1 & 15 & 35 \\
\hline 2 & 5 & 12 \\
\hline 3 & 3 & 7 \\
\hline 4 & 2 & 5 \\
\hline 5 & 0 & 0 \\
\hline 6 & 1 & 2 \\
\hline \multicolumn{3}{|l|}{ Type of outcome $(n=48)$} \\
\hline Pain & 4 & 8 \\
\hline Generic quality of life & 2 & 4 \\
\hline Disease-specific quality of life & 14 & 29 \\
\hline Treatment success & 4 & 8 \\
\hline Other patient-reported outcome & 8 & 17 \\
\hline Mortality, composite including mortality & 2 & 4 \\
\hline Process measure & 8 & 17 \\
\hline Functional measure & 6 & 13 \\
\hline $\begin{array}{l}\text { Valid observations }(n=26)^{\mathrm{a}} \text {, median } \\
\text { (interquartile range), range }\end{array}$ & \multicolumn{2}{|c|}{$127(78-435), 24-4752$} \\
\hline
\end{tabular}

${ }^{\mathrm{a}}$ For studies with more than one primary outcome, the maximum available number of observations was used 
Table 5 Statistical analysis methodology ( $n=43$ unless otherwise stated)

\begin{tabular}{lll}
\hline & Number & Percentage \\
\hline Analysis groups according to randomised groups & & \\
Yes & 42 & 98 \\
No & 1 & 2 \\
Unclear & 0 & 0 \\
Analysis adjusting for non-compliance carried out & \\
Yes & 8 & 19 \\
No & 35 & 81 \\
Unclear & 0 & 0 \\
Compliance analysis type & \\
As treated groups & 3 & 7 \\
Intervention completer subset analysis & 2 & 5 \\
(full compliance) & & \\
Intervention completer subset analysis & 2 & 5 \\
(partial and full compliance) & & \\
Randomisation-based analysis \\
(method unclear) \\
N/A
\end{tabular}

N/A not available

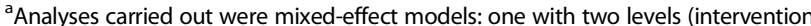
deliverer and) and one with three (site, intervention deliverer, and participant). ${ }^{\mathrm{b}} \mathrm{In}$ one study, the sample size was adjusted to account for clustering by using an intracluster correlation coefficient (ICC) of 0.02; for the other study the observed ICC of the higher level(s) was reported for the two primary outcomes (retention and engagement) under a two level (therapist and participant) and a 3 level model (site, therapist and participant) as 0.180 and 0.22 and 0.099 and 0.110 respectively

Reporting of general study methodology such as randomisation was suboptimal, as has been repeatedly shown for RCTs of both pharmacological and nonpharmacological interventions [17]. Blinding of the primary outcome assessment and of participation was not typically performed, perhaps reflecting the nature of the trial comparison and the types of interventions under evaluation. Studies appeared to adopt a more pragmatic approach to study design [2]. Only two studies adjusted the analysis for clustering of outcome by interventionalist, even though failure to do this could lead to an overly precise estimate, and no studies provided justification for why this was not done [7, 18].

A number of potential advantages have been proposed to support the use of an expertise-based trial design, including addressing the learning curve and possible differences in expertise among health professionals between interventions (sometimes referred to as differential expertise bias, which is particularly likely if a new intervention is compared with an older one), ethical concerns of intervention deliverers about performing a procedure they are not experienced or comfortable with, reassurance for patients that their intervention will be delivered by someone with appropriate 'expertise', improved compliance, avoidance of systematic bias introduced unconsciously if a preference for one intervention exists, and increased participation of interventionalists [2, 3]. However, a number of potential drawbacks have also been noted: the feasibility of two different interventionalists being available, imbalances between interventionalist groups in terms of ability and experience, the need for third-party recruitment, and reduced applicability of the study results $[2,4,6,15,19]$. This review generally reinforced the proposed advantages and disadvantages but highlighted that some of the advantages are conditional on the implementation of the expertise-based design regarding definition of "expertise" (for example, elimination learning). Additional disadvantages were identified, such as the possibility of an expectation bias being created if a new intervention is delivered by a different individual [20], having different individuals recruiting and delivering the intervention which can lead to disagreement regarding suitability for receiving the intervention [21], and the possibility that the intervention may differ in ways beyond the treatment allocation $[20,22]$.

There were a number of limitations to this review. Owing to the inconsistency of reporting highlighted in included studies, it seems likely that some eligible expertise-based trials were not identified, as reference to this aspect of the design was not made in the title or abstracts or possibly anywhere in the text. Inclusion was restricted to two-arm individually randomised parallel group trials, although a form of expertise-based design could be used in other settings (for example, three-arm or cluster RCT). Additionally, the search was conducted only up to 2012. Owing to the small numbers of included studies, it was not possible to formally compare subtypes of trials as planned. Finally, no formal comparison has been made with conventional (within-health professional) trials.

This review expands the current understanding of expertise-based trials. Further work exploring the view and experience of health professionals and those involved in trial design should be carried out to further increase understanding of the decision-making process regarding design choice. We recommend improved and more consistent reporting, and reports should state explicitly who delivered the interventions; the term 'expertise-based' could be used in the title or abstract of trial reports with explicit details regarding the number of interventionalists in each arm with any criteria for eligibility reported in the trial report. 


\section{Conclusions}

Although the use of an expertise-based trial design is growing, it remains uncommon. An expertise-based trial design is an option that should be considered by trials, particularly in light of the high level of recruitment to target and compliance with allocation observed [23]. Its value appears to be context-specific, particularly where interventions differ substantially or interventions are typically delivered by different health professionals. Reporting of expertise-based trials is suboptimal in providing details relating to the expertise design and requires improvement.

\section{Additional files}

Additional file 1: 'Additional file 1-Search Strategies.docx', Search strategies, Database search strategies.

Additional file 2: 'Additional file 1-Search Strategies.docx', Included studies, List of included studies.

\section{Abbreviations}

EMBASE: Excerpta Medica dataBASE; IQR: interquartile range; MEDLINE: Medical Literature Analysis and Retrieval System Online; $\mathrm{RCT}$ : randomised controlled trial.

\section{Competing interests}

The authors declare that they have no competing interests.

\section{Authors' contributions}

JAC had the original idea, wrote the first draft of this article, and helped to devise the search strategy and to undertake the abstract screening, full-text assessment, and data abstraction. CRR also contributed to the original idea and also to the design of the study. DGA and IB contributed to the design of the study. GSM contributed to the design of the study and helped to undertake the abstract screening, full-text assessment, and data abstraction. CF helped to devise the search strategy. $A E, C B$, and TB helped to undertake the abstract screening, full-text assessment, and data abstraction. All authors read and approved the final manuscript.

\section{Acknowledgements}

JAC held a Medical Research Council UK methodology (G1002292) fellowship, which supported this research. The Health Services Research Unit, Institute of Applied Health Sciences (University of Aberdeen), is core-funded by the Chief Scientist Office of the Scottish Government Health and Social Care Directorates. Views express are those of the authors and do not necessarily reflect the views of the funders.

\section{Author details}

${ }^{1}$ Centre for Statistics in Medicine, Nuffield Department of Orthopaedics, Rheumatology and Musculoskeletal Sciences, Botnar Research Centre, University of Oxford, Nuffield Orthopaedic Centre, Windmill Road, Oxford OX3 7LD, UK. ${ }^{2}$ Health Services Research Unit, University of Aberdeen, Health Sciences Building, Foresterhill, Aberdeen AB25 2ZD, UK. ${ }^{3}$ Centre d'Epidémiologie Clinique, Université Descartes, Hôpital Hôtel Dieu, 1 Place du Parvis Notre Dame, Paris, 75004, France.
}

Received: 12 January 2015 Accepted: 5 May 2015

Published online: 30 May 2015

\section{References}

1. Cook JA. The challenges faced in the design, conduct and analysis of surgical randomised controlled trials. Trials. 2009;10:9.

2. Devereaux PJ, Bhandari M, Clarke M, Montori VM, Cook DJ, Yusuf S, et al. Need for expertise based randomised controlled trials. BMJ. 2005;330:88.

3. van der Linden W. Pitfalls in randomized surgical trials. Surgery. 1980;87:258-62.
4. Biau DJ, Porcher R. Letter to the editor re: Orthopaedic surgeons prefer to participate in expertise-based randomized trials. Orthopaedic surgeons prefer to participate in expertise-based randomized trials. Clin Orthop Relat Res. 2009;467:298-300.

5. Walter SD, Ismaila AS, Devereaux PJ, SPRINT Study Investigators. Statistical issues in the design and analysis of expertise-based randomized clinical trials. Stat Med. 2008;27:6583-96.

6. Bednarska E, Bryant D, Devereaux PJ, Expertise-Based Working Group. Orthopaedic surgeons prefer to participate in expertise-based randomized trials. Clin Orthopaed Rel Res. 2008;466:1734-44.

7. Cook JA, Bruckner T, MacLennan GS, Seiler CM. Clustering in surgical trials_database of intracluster correlations. Trials. 2012;13:2.

8. Ergina PL, Cook JA, Blazeby JM, Boutron I, Clavien PA, Reeves BC, et al. Challenges in evaluating surgical innovation. Lancet. 2009;374:1097-104.

9. Mastracci TM, Clase CM, Devereaux PJ, Cinà CS. Open versus endovascular repair of abdominal aortic aneurysm: a survey of Canadian vascular surgeons. Can J Surg. 2008;51:142-8.

10. Roberts C, Roberts SA. Design and analysis of clinical trials with clustering effects due to treatment. Clin Trials. 2005;2:152-62.

11. Vierron E, Giraudeau B. Design effect in multicenter studies: gain or loss of power? BMC Med Res Methodol. 2009;9:39.

12. Walwyn $R$, Roberts $C$. Therapist variation within randomised trials of psychotherapy: implications for precision, internal and external validity. Stat Methods Med Res. 2010;19:291-315.

13. Cook JA, Ramsay CR, Norrie J. Recruitment to publicly funded trials-are surgical trials really different? Contemporary Clin Trials. 2008;29:631-4.

14. Scholtes VA, Nijman TH, van Beers L, Devereaux PJ, Poolman RW. Emerging designs in orthopaedics: expertise-based randomized controlled trials. J Bone Joint Surg Am. 2012;94:8.

15. Rudicel S, Esdaile J. The randomized clinical trial in orthopaedics: obligation or option? J Bon Joint Surg Am. 1985;67:1284-93.

16. Beard D, Price A, Cook J, Fitzpatrick R, Carr A, Campbell M, et al. Total or partial knee arthroplasty trial-TOPKAT: study protocol for a randomised controlled trial. Trials. 2013;14:292.

17. Boutron I, Tubach F, Giraudeau B, Ravaud P. Methodological differences in clinical trials evaluating nonpharmacological and pharmacological treatments of hip and knee osteoarthritis. JAMA. 2003;290:1062-70.

18. Kahan BC, Morris TP. Assessing potential sources of clustering in individually randomised trials. BMC Med Res Methodol. 2013;13:58.

19. Pike K, Angelinin G, Reeves BC, Taggart D, Rogers C. Recruitment challenges in surgical trials: lessons from the crisp trial. Trials. 2013;14 Suppl 1:27.

20. Wilkey A, Gregory M, Byfield D, McCarthy PW. A comparison between chiropractic management and pain clinic management for chronic low-back pain in a national health service outpatient clinic. J Altern Complement Med. 2008;14:465-73.

21. Grant AM, Wileman SM, Ramsay CR, Mowat NA, Krukowski ZH, Heading RC, et al. Minimal access surgery compared with medical management for chronic gastro-oesophageal reflux disease: UK collaborative randomised trial. BMJ. 2009;338:81-3.

22. Alobaid A, Harvey EJ, Elder GM, Lander P, Guy P, Reindl R. Minimally invasive dynamic hip screw. Prospective randomized trial of two techniques of insertion of a standard dynamic fixation device. J Orthop Trauma. 2007;18:207-12.

23. Cook JA, McCulloch P, Blazeby JM, Beard DJ, Marinac-Dabic D, Sedrakyan A, et al. IDEAL framework for surgical innovation 3: randomised controlled trials in the assessment stage and evaluations in the long term study stage. BMJ. 2013;346:f2820. 